\title{
Engagement in care among youth living with parenterally-acquired
}

\section{HIV infection in Romania}

Cosmina Gingaras ${ }^{\mathrm{a} *}{ }^{\ddagger}$, Colette Smith $^{\mathrm{b}}$, Roxana Radoi ${ }^{\mathrm{a}}$, Diana Sima ${ }^{\mathrm{a}}$, Mike Youle $^{\mathrm{c}}$ and Luminita Ene ${ }^{\mathrm{a}}$

a HIV Department, "Dr.V.Babes" Clinical Hospital for Infectious and Tropical Diseases, Bucharest, Romania; ${ }^{b}$ Research Department of Infection and Population Health, University College London, UK; ${ }^{\mathrm{c}}$ HIV Clinical Research, Royal Free Center for HIV Medicine, London, UK

*Corresponding author: cosminagingaras@gmail.com

${ }^{¥}$ These authors contributed equally to this work. 


\section{Engagement in care among youth living with parenterally-acquired HIV infection in Romania}

Transition from adolescent to adult care can be challenging for youth living with HIV. We describe the level of engagement in care and its impact on HIV outcomes in a group of patients infected in early childhood and followed-up through adolescence (15-19 years) and young adulthood (20-24 years) by the same medical team. We conducted a cohort study of youth born between 19851993 and infected with HIV parenterally, followed at a single tertiary care centre. Individuals were followed from age 15 years or first clinic visit (whichever came last) until age 25 years or 30 Nov 2016 (whichever came first). A longitudinal continuum-of-care was constructed, categorizing individuals' status for each month between the ages of 15-25 years as: engaged in care (EIC); not in care (NIC: no clinic visits within past year); lost-to-follow-up (LTFU: NIC and did not return to clinic); or died. Those EIC were further subdivided by current CD4 count and viral load (VL). 545 individuals (52\% male) were followed for 4775 person-years. 64 (12\%) became LTFU and 27 (5\%) died. At age 15, 92\% were

EIC, decreasing to $84 \%$ at age 20 and $74 \%$ at age 25 . Of those EIC, HIV markers improved with age: $79 \%$ and $52 \%$ had a CD $4 \geq 200$ cells $/ \mu 1$ and $\mathrm{VL}<400 \mathrm{cps} / \mathrm{ml}$ at age 15; increasing to $86 \%$ and $73 \%$ at age 20 and $87 \%$ and $80 \%$ at age 25.277 (55.5\%) spent their entire adolescence EIC; this decreased to 202 (37\%) for the years of young adulthood $(\mathrm{p}=0.0001)$. There were no observed demographic differences between those with continuous and intermittent engagement in care. We conclude that youth infected during early childhood tended to disengage from care, even when followed by the same medical team for a lengthy period of time. For those that did engage in care, HIV-related outcomes improved from adolescence through to adulthood.

Keywords: HIV, youth, adolescents, transition of care, engagement in care

\section{Introduction}

In 2016, UNAIDS estimated that 1.8 million adolescents (10-19 years old) were living with HIV and 43,400 deaths were due to HIV in this population, making HIV the 8 th cause of mortality among adolescents worldwide (IeDEA Pediatric Working Group, 2017). In the continuum of care, adolescents and young adults significantly lag behind 
adults in achieving the UNAIDS 90 (diagnosed)- 90 (treated)- 90 (undetectable) goals at every step of this cascade (Zanoni \& Mayer, 2014). Successful transition of adolescents living with HIV is an important public health concern because it impacts disease morbidity, mortality and transmission of infection at an age of increased risk-taking behaviors.

As with other chronic medical conditions, healthcare transition of youth living with HIV is defined as the planned and purposeful movement from child-centered to adult-centered care (Blum et al., 1993). One metric of successful transition is engagement in care. Broadly defined as maintenance of HIV-related healthcare after initial linkage, i.e. a certain level of adherence to scheduled clinic appointments (usually every 3-6 months) (Philbin et al., 2014), engagement in care is associated with improved clinical and virological outcomes and reduced mortality (Minniear et al., 2013; Ulett et al., 2009).

Transition of care is a complex process and there is a growing body of literature investigating the barriers to a successful transition (Judd \& Davies, 2018; Kim, Kim, McDonald, Fidler, \& Foster, 2017; Lolekha et al., 2017; Philbin et al., 2014; Westling, Naver, Vesterbacka, \& Belfrage, 2016). These barriers can be structural (e.g. maintaining healthcare insurance; social stigma), provider-related (e.g. the proactive role of paediatricians in a child's health versus the level of self-sufficiency and responsibility expected at the adult-level provider) or individual (family support, psychosocial and behavioral changes specific to adolescence) (Philbin et al., 2017). Most transition models need to overcome barriers at all of these three levels.

The national program for prevention, surveillance and control of HIV/AIDS in Romania provides HIV care and treatment free of charge. HIV-infected children and their families benefit from the support of medical and psychosocial services free of 
charge and receive a monthly allowance depending on disease severity and disability. In general, youth are required to keep at least yearly clinic appointments to maintain these benefits. In some of the regional centers the same medical team will care for HIVinfected children through adulthood.

The purpose of our study was to explore the extent of engagement and the predictors of disengagement from care among a cohort of youth living with HIV, parenterally infected as children, who remained in the care of the same medical team from childhood into adulthood.

\section{Methods}

\section{Study population}

The "Victor Babes" Clinical Hospital for Infectious and Tropical Diseases, Bucharest $(\mathrm{VBH})$ is one of the nine reference centres for HIV in Romania. The first paediatric case of HIV was diagnosed at VBH in 1989, and ever since, 1638 children have been diagnosed and treated at VBH. Starting in the year 2000, a total of 628 children were enrolled in a prospective cohort study, and data (demographic, clinical, laboratory, treatment) were recorded in electronic format in the VHB paediatric database using a modified HICDEP protocol (www.hicdep.org). Patients from the paediatric cohort, now entering adulthood, are largely followed by the same medical team that cared for them in their childhood. HIV diagnosis was disclosed around 14 years of age, but by 18 , most adolescents knew their HIV status. The standard of care at the clinic consists of CD4 measurement every 3-6 months and HIV viral load at least once a year, although this was not always possible because of financial constraints. Antiretroviral treatment has been in use since 1996 and became widely available in 2000 through the national HIV program. 


\section{Study design}

We conducted a cohort study which included youths followed at VBH, born between 1985-1993 and infected with HIV parenterally in their first years of life. Individuals were followed from age 15 years or first clinic visit (for those whose first visit at our clinic occurred after 15 years) until age 25 years or 30 Nov 2016 (whichever came first). The local VBH ethics committee approved the analysis of anonymized data from these patients.

\section{Definitions of variables}

Adolescence was defined as age 15 to 19 . Young adulthood included ages 20 to 25 .

HIV viral load (VL) was measured by commercially available assays (thresholds for detection varied over time). Participants with HIV RNA $<400$ copies/ml were classified as having undetectable viral load.

Visits were recorded as dates when CD4 count and/or HIV viral load were measured. If a patient presented to the out-patient clinic with a medical issue or was hospitalized, but no CD4 or viral load measurements were taken, these visits were not recorded. However, it is very likely that during the clinic encounter, if the medical issue prompted it and/or if more than 6 months had passed since the previous assessment, a CD4 count or viral load measurement were taken, and the visit was recorded.

Engaged in care (EIC): having at least one visit in the previous year. Each month that a patient was engaged in care was added to their total time of engagement in care.

Not in care (NIC): no clinic visits within the past year, but returned to the clinic within the study period. Each month that the patient exceeded one year since his previous visit was added to their total time of non-engagement in care.

Lost to follow-up (LTFU): no clinic visits within the past year and did not return to the clinic within the study period. 


\section{Statistical analysis}

A longitudinal continuum-of-care was constructed, following a similar method (Howarth et al., 2017). Each individual's follow-up was divided into one-month periods, and defined according to their current age for that month. Each individuals' status for each month of follow-up was then categorized as: engaged in care; not in care; lost-to-follow-up; or dead.

We calculated the percentage of young people in each category according to current age, across the ages of 15 to 25 years and the results were diagrammed using a xxx plot. We particularly focused on the percentages in each Engagement In Care category at the ages of 15, 20 and 25 years. Those EIC were further subdivided by current CD4 ( $<200$ vs $\geq 200$ cells $/ \mu 1$ ) and current VL (detectable vs undetectable). The cumulative person-time spent in each Engagement In Care category according to several demographic factors (gender, HBV and HCV status, previous AIDS diagnosis, current age, age at HIV diagnosis, CD4\% nadir, latest CD4 count, undetectable viral load, and ART status) was calculated and compared using Poisson regression with Generalised Estimating Equations. In addition, for each individual, we calculated the percentage of follow-up months that they were engaged in care. The characteristics of those that remained EIC were compared to those that did not. Analyses were performed using SAS Version 9.4 (SAS Institute Inc, Cary, NC).

\section{Results}

A total of 545 people was included in the present study and followed for 4775 personyears; out of these, $313(57 \%)$ were followed for the entire 10 years. At the end of the study period, $66(12.1 \%)$ were LTFU and 27 (1.3\%) died.

Table 1 shows a summary of demographic, virological, immunological, therapeutic and coinfection data. More than half were male and the median age at 
diagnosis was 7.4 years. A third of patients had a documented AIDS-defining disease, almost a third had a positive hepatitis B antigen serology but very few had evidence of HCV infection. Most patients benefited from antiretroviral treatment, for a median duration of 8 years, and with an equal exposure to protease inhibitors (PIs) and nonnucleoside reverse transcription inhibitors (NNRTIs). Out of 260 women included in the study, 61 (23.4\%) became pregnant and delivered 82 children (44 had one child, 13 had two children, four had three children during the study period), none of whom were infected with HIV.

Over half of patients $(55.5 \%, \mathrm{~N}=277)$ maintained perfect engagement in care (100\% of follow-up time) throughout adolescence. However, during the years of young adulthood, engagement in care dropped significantly to $37 \%(\mathrm{~N}=202)(\mathrm{p}=0.0001)$.

At study entry (age 15 or first visit at VBH after this age), 92\% of patients were EIC; $8 \%$ were NIC/LFTU (deaths were not recorded). At the beginning of young adulthood (age 20): $84 \%$ were EIC; $15 \%$ were NIC/LFTU and $0.1 \%$ were dead. At the end of the study (the first of age 25 or November 30th, 2016): 74\% were EIC, $22 \%$ were NIC/LFTU and $4 \%$ deceased.

Of those engaged in care, objective parameters of HIV infection improved over time, as shown in Figure 1. At age 15, 79\% of patients had CD4>200 cells/ul, increasing to $86 \%$ at age 20 , and $87 \%$ at age 25 . Similarly, VL was undetectable in $19 \%$ at 15 years of age, which increased to $45 \%$ at age 20 , and to $61 \%$ at age 25 . The percentages on ART at the same ages were $89 \%, 88 \%$ and $86 \%$ respectively. Looking at AIDS diagnoses within the previous 6 months, this metric also improved over time from $10 \%$ at age 15 , to $5 \%$ at 20 , and $3 \%$ at 25 .

The cumulative person-time engaged in care was compared to cumulative person-time not-engaged in care by different relevant predictors (see Table 2). Last CD4 
count and last VL measurement were significantly associated with disengagement from care, those with severe immunosuppression and detectable viral load having higher odds of disengagement.

\section{Discussion}

Our study shows that youth infected with HIV during early childhood tend to disengage from care even when they are followed by the same medical team for a lengthy period. However, for those who remained engaged in care, objective markers of HIV disease (e.g. CD4 count, viral load, AIDS-defining illnesses) improved from adolescence through adulthood.

The HIV epidemic in Romania in the 1990s was mainly driven by the parenteral transmission of HIV in children. It was estimated that 10,000 children acquired HIV nosocomially between 1986 and 1992 through reuse of needles and lack of testing of blood and blood-products (Buzducea, Lazar, \& Mardare, 2010). Almost 3,000 of these children died, however many survived and are now in their late 20s (Buzducea et al., 2010). By the end of 2016, almost 6,500 HIV-infected persons 20-29 years old were alive, most of them infected during the Romanian HIV paediatric epidemic (The National Institute of Infectious Diseases 'Prof. Dr. Matei Bals', 2016). Antiretroviral treatment became available in Romania in 1996 and starting in 2000 combination antiretroviral therapy was available through the national HIV program. Our study population had almost lifelong HIV infection, similar to perinatally-infected children; however, in comparison, they did not benefit from early diagnosis and treatment because of social and political changes around the time the epidemic became a public health problem.

Our clinic model of continued medical care of children through their adolescence and adulthood, while not unique among low and middle-income countries 
(Judd \& Davies, 2018), allows for a separation of the effect of structural and provider barriers from individual-level difficulties (physical, psychological, behavioral) in the context of chronic infection.

Adolescence is a developmental stage characterized by immature thought processes, impulsivity, increased risk-taking behaviors (such as unprotected sex or substance abuse), and vulnerability to peer-pressure (Hosek \& Zimet, 2010). youth living with HIV suffer from additional adversity that comes with managing a chronic disease, social stigma and discrimination, neurocognitive dysfunction (Ene et al., 2014; Ruel et al., 2012), physical deformity resulting from opportunistic infections or medication side-effects (S. Lee \& Hazra, 2015; Vreeman, McCoy, \& Lee, 2017). Depression and anxiety are more prevalent in perinatally-infected adolescents compared to HIV-negative peers (Mellins \& Malee, 2013) and have been associated with lower adherence to ART and consequently poorer virological outcomes (Kang, Delzell, Chhabra, \& Oberdorfer, 2015; Murphy et al., 2001; Williams et al., 2006).

A successful transition from adolescent to adult care is at the very least an improvement in immunological and virological outcomes (Tepper, Zaner, \& Ryscavage, 2017; Zanoni \& Mayer, 2014). In our study we found that among those engaged in care, the percent of virologically suppressed and the percent of non-severe immunosuppressed increased over time from adolescence to young adulthood, and the same beneficial trend was observed for the percent of new AIDS diagnoses. These results suggest that early in adolescence adherence to treatment may be poorer compared to adulthood or they reflect access to better antiretroviral regimens (simpler, more tolerable, more potent). Or this improvement of HIV markers in young adulthood can be explained by behavioral changes, assuming adult responsibilities and "normalization" of life with HIV. Even if the rate of unemployment is high (Luca, 
Lazar, Luca, Ene, \& Achim, 2016), youth living with HIV became independent and started their own families. One quarter of the women became pregnant during the observation period and none of the babies were infected. This reflects good retention in care and optimal adherence to ART in these women, at the very least when faced with the responsibility for the health of their unborn children.

The profile of youth living with HIV NIC is suggestive of non-adherence, with detectable HIV viral load and low nadir CD4. We speculate that treatment fatigue and late disclosure of HIV diagnosis are important contributors to this profile. Pill burden in children and increased autonomy in adolescence have been cited as significant predictors of treatment fatigue and consequently declining adherence over time (Claborn, Meier, Miller, \& Leffingwell, 2015). Although ART coverage was high at three time points (over 85\%), this reflects prescription of ART rather than adherence to it.

Early disclosure of HIV diagnosis has been associated with safer sexual behavior, increased responsibility over one's health, and in some reports -but not allwith improved adherence to treatment (Gyamfi, Okyere, Appiah-Brempong, Adjei, \& Mensah, 2015). A review of diagnosis disclosure to children which included studies from 1996 to 2011 found that children from low- and middle-income countries were being disclosed to later (median 9.6 years, range 8.1-15) and the rate of disclosure was only $20 \%$ compared to $43 \%$ in high-income countries (Pinzon-Iregui, Beck-Sague, \& Malow, 2013). In our cohort, children were disclosed to around 14 years of age, but by 18, most adolescents knew their diagnosis. We expect that this late disclosure negatively impacted adolescents' engagement in care, a hypothesis we could not formally test because we did not capture this variable in our dataset. 
The rate of engagement in care in our study was relatively high but declined over time. Our approach to measuring engagement in care in a cohort of children followed over time to adulthood at the same clinic is novel, making comparisons to other studies difficult. If we consider age at transition 17-18 years (or later, at 22 years), then we can attempt to compare our results at the 20 years time point (or 25 years for later transition ages) to studies reporting retention in care at approximately 2 years posttransition from adolescent to adult care. We thus observed similar or higher retention in care in our model (Hussen et al., 2015; Izzo et al., 2018; Kakkar et al., 2016; Ryscavage, Macharia, Patel, Palmeiro, \& Tepper, 2016), suggesting the importance of continuity of care or of a smooth transition process.

The rate of LTFU (12.1\%) was also lower or similar to other reports (Sainz \& Navarro, 2017; Weijsenfeld et al., 2016). A recent pooled analysis of twelve cohorts from the Collaborative Initiative for Pediatric HIV Education and Research (CIPHER) which included adolescents from around the world from 10 years of age and measured outcomes at 15 years of age, reported an overall cumulative LTFU rate of $11.3 \%$ (Collaborative Initiative for Paediatric HIV Education and Research (CIPHER) Global Cohort Collaboration et al., 2018).

Our study has several limitations. First, our definition of engagement in care was laxer than in other studies (L. Lee et al., 2016; Philbin et al., 2016). Although we allowed for a longer period between clinic appointments in our definition of engagement in care, these were usually only visits for CD4 count and HIV viral load assessment and there were likely clinic and medication visits in between that were not recorded in our database. Therefore, we expect that our results are comparable to studies that used stricter definitions of engagement in care. Second, the period effect, i.e. changing trends in treatment may explain the improved virological and immunological 
outcomes in youth, irrespective of level of engagement in care. Third, important predictors for engagement in care were not measured, such as mental health indicators (depression, anxiety), cognitive factors (degrees of cognitive deficits), and social factors (orphan, living in foster home, housing, insurance, income, disclosure of HIV status).

In conclusion, in a large cohort of parenterally-infected children followed by the same medical team, the rate of disengagement from care increased from adolescence to youth, with a quarter of patients being disengaged after almost 10 years of follow-up. Although retention in care was higher than that reported in studies of formal transition programs from paediatric to adult care at 18 or 21 years of age, it is likely that the steady time trend towards disengagement would be even steeper in our population if care was discontinued at those young ages. Last, patients who remained engaged in care had better clinical outcomes, suggesting that successful transitions happen at older ages and require an adequate preparation time.

\section{Disclosure statement}

No potential conflict of interest was reported by the authors.

\section{References}

Blum, R. W., Garell, D., Hodgman, C. H., Jorissen, T. W., Okinow, N. A., Orr, D. P., \& Slap, G. B. (1993). Transition from child-centered to adult health-care systems for adolescents with chronic conditions. A position paper of the society for adolescent medicine. The Journal of Adolescent Health : Official Publication of the Society for Adolescent Medicine, 14(7), 570-576. doi:1054-139X(93)90143-D [pii] 
Buzducea, D., Lazar, F., \& Mardare, E. I. (2010). The situation of romanian HIVpositive adolescents: Results from the first national representative survey. AIDS Care, 22(5), 562-569. doi:10.1080/09540120903280927 [doi]

Claborn, K. R., Meier, E., Miller, M. B., \& Leffingwell, T. R. (2015). A systematic review of treatment fatigue among HIV-infected patients prescribed antiretroviral therapy. Psychology, Health \& Medicine, 20(3), 255-265.

doi:10.1080/13548506.2014.945601 [doi]

Collaborative Initiative for Paediatric HIV Education and Research (CIPHER) Global Cohort Collaboration, Slogrove, A. L., Schomaker, M., Davies, M. A., Williams, P., Balkan, S., .. . Leroy, V. (2018). The epidemiology of adolescents living with perinatally acquired HIV: A cross-region global cohort analysis. PLoS Medicine, 15(3), e1002514. doi:10.1371/journal.pmed.1002514 [doi]

Ene, L., Franklin, D. R., Burlacu, R., Luca, A. E., Blaglosov, A. G., Ellis, R. J., . . Marcotte, T. D. (2014). Neurocognitive functioning in a romanian cohort of young adults with parenterally-acquired HIV-infection during childhood. Journal of Neurovirology, 20(5), 496-504. doi:10.1007/s13365-014-0275-1 [doi]

Gyamfi, E., Okyere, P., Appiah-Brempong, E., Adjei, R. O., \& Mensah, K. A. (2015). Benefits of disclosure of HIV status to infected children and adolescents: Perceptions of caregivers and health care providers. The Journal of the Association of Nurses in AIDS Care : JANAC, 26(6), 770-780. doi:10.1016/j.jana.2015.08.001 [doi] 
Hosek, S. G., \& Zimet, G. D. (2010). Behavioral considerations for engaging youth in HIV clinical research. Journal of Acquired Immune Deficiency Syndromes (1999), 54 Suppl 1, S25-30. doi:10.1097/QAI.0b013e3181e15c22 [doi]

Howarth, A. R., Burns, F. M., Apea, V., Jose, S., Hill, T., Delpech, V. C., . . UK Collaborative HIV Cohort (UK CHIC) study. (2017). Development and application of a new measure of engagement in out-patient HIV care. HIV Medicine, 18(4), 267-274. doi:10.1111/hiv.12427 [doi]

Hussen, S. A., Chahroudi, A., Boylan, A., Camacho-Gonzalez, A. F., Hackett, S., \& Chakraborty, R. (2015). Transition of youth living with HIV from pediatric to adult-oriented healthcare: A review of the literature. Future Virology, 9(10), 921929. doi:10.2217/fvl.14.73 [doi]

IeDEA Pediatric Working Group. (2017). Taking a critical look at the UNAIDS global estimates on paediatric and adolescent HIV survival and death. Journal of the International AIDS Society, 20(1), 21952. doi:10.7448/IAS.20.1.21952 [doi]

Izzo, I., Quiros-Roldan, E., Saccani, B., Chiari, E., Casari, S., Foca, E., . . Castelli, F. (2018). Perinatally HIV-infected youths after transition from pediatric to adult care, a single-center experience from northern italy. AIDS Research and Human Retroviruses, 34(3), 241-243. doi:10.1089/AID.2017.0120 [doi]

Judd, A., \& Davies, M. A. (2018). Adolescent transition among young people with perinatal HIV in high-income and low-income settings. Current Opinion in HIV and AIDS, doi:10.1097/COH.0000000000000448 [doi] 
Kakkar, F., Van der Linden, D., Valois, S., Maurice, F., Onnorouille, M., Lapointe, N., . . Lamarre, V. (2016). Health outcomes and the transition experience of HIVinfected adolescents after transfer to adult care in quebec, canada. BMC Pediatrics, 16, 109-016-0644-4. doi:10.1186/s12887-016-0644-4 [doi]

Kang, E., Delzell, D. A., Chhabra, M., \& Oberdorfer, P. (2015). Factors associated with high rates of antiretroviral medication adherence among youth living with perinatal HIV in thailand. International Journal of STD \& AIDS, 26(8), 534-541. doi:10.1177/0956462414545524 [doi]

Kim, S., Kim, S. H., McDonald, S., Fidler, S., \& Foster, C. (2017). Transition to adult services - a positive step. AIDS Care, 29(7), 885-889. doi:10.1080/09540121.2016.1268672 [doi]

Lee, L., Yehia, B. R., Gaur, A. H., Rutstein, R., Gebo, K., Keruly, J. C., . . HIV Research Network. (2016). The impact of youth-friendly structures of care on retention among HIV-infected youth. AIDS Patient Care and STDs, 30(4), 170177. doi:10.1089/apc.2015.0263 [doi]

Lee, S., \& Hazra, R. (2015). Achieving 90-90-90 in paediatric HIV: Adolescence as the touchstone for transition success. Journal of the International AIDS Society, 18(Suppl 6), 20257. doi:10.7448/IAS.18.7.20257 [doi]

Lolekha, R., Boon-Yasidhi, V., Na-Nakorn, Y., Manaboriboon, B., Vandepitte, W. P., Martin, M., . . Chokephaibulkit, K. (2017). The happy teen programme: A holistic outpatient clinic-based approach to prepare HIV-infected youth for the transition from paediatric to adult medical care services in thailand. Journal of the International AIDS Society, 20(Suppl 3), 21500. doi:10.7448/IAS.20.4.21500 [doi] 
Luca, A., Lazar, F., Luca, A. E., Ene, L., \& Achim, C. (2016). Employment status controls the relationship between neurocognitive impairment and depression in a cohort of young HIV-infected adults since childhood [Abstract]. BMC Infectious Diseases, 16 Retrieved from https://bmcinfectdis.biomedcentral.com/articles/10.1186/s12879-016-1480-

\section{$\underline{8 \# \operatorname{Sec} 33}$}

Mellins, C. A., \& Malee, K. M. (2013). Understanding the mental health of youth living with perinatal HIV infection: Lessons learned and current challenges. Journal of the International AIDS Society, 16, 18593. doi:10.7448/IAS.16.1.18593 [doi]

Minniear, T. D., Gaur, A. H., Thridandapani, A., Sinnock, C., Tolley, E. A., \& Flynn, P. M. (2013). Delayed entry into and failure to remain in HIV care among HIVinfected adolescents. AIDS Research and Human Retroviruses, 29(1), 99-104. doi:10.1089/AID.2012.0267 [doi]

Murphy, D. A., Wilson, C. M., Durako, S. J., Muenz, L. R., Belzer, M., \& Adolescent Medicine HIV/AIDS Research Network. (2001). Antiretroviral medication adherence among the REACH HIV-infected adolescent cohort in the USA. AIDS Care, 13(1), 27-40. doi:10.1080/09540120020018161 [doi]

Philbin, M. M., Tanner, A. E., Chambers, B. D., Ma, A., Ware, S., Lee, S., . . The Adolescent Trials, N. (2017). Transitioning HIV-infected adolescents to adult care at 14 clinics across the united states: Using adolescent and adult providers' insights to create multi-level solutions to address transition barriers. AIDS Care, 29(10), 1227-1234. doi:10.1080/09540121.2017.1338655 [doi] 
Philbin, M. M., Tanner, A. E., DuVal, A., Ellen, J. M., Xu, J., Kapogiannis, B., . . Adolescent Trials Network for HIV/AIDS Interventions. (2014). Factors affecting linkage to care and engagement in care for newly diagnosed HIV-positive adolescents within fifteen adolescent medicine clinics in the united states. AIDS and Behavior, 18(8), 1501-1510. doi:10.1007/s10461-013-0650-6 [doi]

Philbin, M. M., Tanner, A. E., DuVal, A., Ellen, J. M., Xu, J., Kapogiannis, B., . . . Adolescent Trials Network for HIVAIDS Interventions. (2016). HIV testing, care referral, and linkage to care intervals affect time to engagement in care for newly diagnosed HIV-infected adolescents in 15 adolescent medicine clinics in the united states. Journal of Acquired Immune Deficiency Syndromes (1999), 72(2), 222-229. doi:10.1097/QAI.0000000000000958 [doi]

Pinzon-Iregui, M. C., Beck-Sague, C. M., \& Malow, R. M. (2013). Disclosure of their HIV status to infected children: A review of the literature. Journal of Tropical Pediatrics, 59(2), 84-89. doi:10.1093/tropej/fms052 [doi]

Ruel, T. D., Boivin, M. J., Boal, H. E., Bangirana, P., Charlebois, E., Havlir, D. V., . . Wong, J. K. (2012). Neurocognitive and motor deficits in HIV-infected ugandan children with high CD4 cell counts. Clinical Infectious Diseases : An Official Publication of the Infectious Diseases Society of America, 54(7), 1001-1009. doi:10.1093/cid/cir1037 [doi]

Ryscavage, P., Macharia, T., Patel, D., Palmeiro, R., \& Tepper, V. (2016). Linkage to and retention in care following healthcare transition from pediatric to adult HIV care. AIDS Care, 28(5), 561-565. doi:10.1080/09540121.2015.1131967 [doi] 
Sainz, T., \& Navarro, M. L. (2017). HIV-infected youths: Transition in spain compared to the netherlands. Clinical Infectious Diseases : An Official Publication of the Infectious Diseases Society of America, 64(2), 230. doi:10.1093/cid/ciw702 [doi]

Tepper, V., Zaner, S., \& Ryscavage, P. (2017). HIV healthcare transition outcomes among youth in north america and europe: A review. Journal of the International AIDS Society, 20(Suppl 3), 21490. doi:10.7448/IAS.20.4.21490 [doi]

The National Institute of Infectious Diseases 'Prof. Dr. Matei Bals'. (2016). Evolution of HIV infection in romania. Retrieved from http://www.cnlas.ro/images/doc/01122016.pdf

Ulett, K. B., Willig, J. H., Lin, H. Y., Routman, J. S., Abroms, S., Allison, J., . . . Mugavero, M. J. (2009). The therapeutic implications of timely linkage and early retention in HIV care. AIDS Patient Care and STDs, 23(1), 41-49. doi:10.1089/apc.2008.0132 [doi]

Vreeman, R. C., McCoy, B. M., \& Lee, S. (2017). Mental health challenges among adolescents living with HIV. Journal of the International AIDS Society, 20(Suppl 3), 21497. doi:10.7448/IAS.20.4.21497 [doi]

Weijsenfeld, A. M., Smit, C., Cohen, S., Wit, F. W. N. M., Mutschelknauss, M., van der Knaap, L. C., . . Kortmann, W. (2016). Virological and social outcomes of HIVinfected adolescents and young adults in the netherlands before and after transition to adult care. Clinical Infectious Diseases : An Official Publication of the Infectious Diseases Society of America, 63(8), 1105-1112. doi:10.1093/cid/ciw487 [doi] 
Westling, K., Naver, L., Vesterbacka, J., \& Belfrage, E. (2016). Transition of HIVinfected youths from paediatric to adult care, a swedish single-centre experience. Infectious Diseases (London, England), 48(6), 449-452. doi:10.3109/23744235.2016.1143964 [doi]

Williams, P. L., Storm, D., Montepiedra, G., Nichols, S., Kammerer, B., Sirois, P. A., . . . PACTG 219C Team. (2006). Predictors of adherence to antiretroviral medications in children and adolescents with HIV infection. Pediatrics, 118(6), e1745-57. doi:peds.2006-0493 [pii]

Zanoni, B. C., \& Mayer, K. H. (2014). The adolescent and young adult HIV cascade of care in the united states: Exaggerated health disparities. AIDS Patient Care and STDs, 28(3), 128-135. doi:10.1089/apc.2013.0345 [doi] 
Table 1. Characteristics of study participants $(\mathrm{N}=545)$.

Gender

HBs Ag positive

HCV Ab positive

Ever AIDS diagnosis

Age at HIV diagnosis

Age at first visit at VBH

Latest VL $<400$ copies/ml

Latest CD4 absolute count

CD4 nadir absolute count

CD4 nadir percentage

Years since ART initiated $(\mathrm{N}=505)$

Ever received NNRTI

Ever received PI

Ever received INSTI
Male

$285(52.3 \%)$

Yes

Unknown

Yes

Unknown

Yes

Median (IQR)

Median (IQR)

Yes

Median (IQR)

Median (IQR)

Median (IQR)

Median

Yes

Yes

Yes
$186(34.5 \%)$

$415(77.0 \%)$

$413(76.6 \%)$

$175(32.7 \%)$

$1(0.2 \%)$

$17(3.2 \%)$

$1(0.2 \%)$

$7.4(4.5,11.3)$

$11.6(7.4,15.3)$

$336 / 344(66.8 \%)$

$504(328,701)$

$83(19,221)$

$8(3,15)$

8.0

$24(4.5 \%)$ 
Table 2. Predictors of disengagement from care

\begin{tabular}{|c|c|c|c|c|}
\hline & & $\begin{array}{r}\text { Total } \\
\text { person- } \\
\text { years }\end{array}$ & $\begin{array}{r}\text { Time spent NIC } \\
(\%)\end{array}$ & P-value \\
\hline Total follow-up & & 4115 & $525(12.8 \%)$ & \\
\hline \multirow{2}{*}{ Gender } & Male & 2185.49 & $279.39(12.8)$ & 0.19 \\
\hline & Female & 1929.71 & $245.81(12.7)$ & \\
\hline \multirow[t]{2}{*}{ HBs Ag positive } & No/Unknown & 2787.14 & $361.74(13.0)$ & 0.61 \\
\hline & Yes & 1328.06 & $163.47(12.3)$ & \\
\hline \multirow[t]{2}{*}{ HCV Ab positive } & No/Unknown & 3974.76 & 491.95 (12.4) & 0.10 \\
\hline & Yes & 140.44 & $33.26(23.7)$ & \\
\hline \multirow{2}{*}{ Ever AIDS diagnosis } & No & 2387.81 & $310.77(13.0)$ & 0.82 \\
\hline & Yes & 1727.38 & $214.44(12.4)$ & \\
\hline \multirow[t]{5}{*}{ Age at first visit at VBH (years) } & $0<10$ & 1746.40 & $211.70(12.1)$ & 0.12 \\
\hline & $110-12$ & 665.39 & $77.36(11.6)$ & \\
\hline & $213-15$ & 892.52 & $109.68(12.3)$ & \\
\hline & $316-18$ & 495.08 & $81.63(16.5)$ & \\
\hline & $419+$ & 315.81 & $44.83(14.2)$ & \\
\hline \multirow[t]{6}{*}{ Age at diagnosis (years) } & $0<2$ & 361.31 & $50.76(14.1)$ & 0.14 \\
\hline & $12-4$ & 809.99 & $118.60(14.6)$ & \\
\hline & $25-9$ & 1665.63 & 195.37 (11.7) & \\
\hline & $310-15$ & 954.04 & 121.14 (12.7) & \\
\hline & $416+$ & 324.23 & & \\
\hline & & & $39.33(12.1)$ & \\
\hline \multirow{4}{*}{ CD4 \% nadir } & $0<5$ & 1422.62 & 170.67 (12.0) & 0.64 \\
\hline & $15-9$ & 890.10 & $104.58(11.8)$ & \\
\hline & $210-19$ & 1264.71 & $163.60(12.9)$ & \\
\hline & $320+$ & 537.77 & $86.36(16.1)$ & \\
\hline \multirow[t]{4}{*}{ Last CD4 count (cells/mm $\left.{ }^{3}\right)$} & $0<\mathbf{2 0 0}$ & 553.66 & $83.83(15.1)$ & $<0.0001$ \\
\hline & $1200-349$ & 648.94 & 101.00 (15.6) & \\
\hline & $2350-499$ & 818.89 & $97.30(11.9)$ & \\
\hline & $3500+$ & 2093.70 & 243.07 (11.6) & \\
\hline \multirow[t]{2}{*}{$\mathrm{Vl}<\mathbf{4 0 0}$ copies $/ \mathrm{ml}$} & No & 1102.27 & 208.64 (18.9) & $<0.0001$ \\
\hline & Yes & 3012.93 & $316.56(10.5)$ & \\
\hline \multirow[t]{5}{*}{ Total time on ART (years) } & 0 Not on ART & 102.99 & $42.12(40.9)$ & 0.08 \\
\hline & $10-5$ & 551.56 & $53.28(9.7)$ & \\
\hline & $25-10$ & 1507.41 & 200.64 (13.3) & \\
\hline & $310-15$ & 1497.53 & $182.41(12.2)$ & \\
\hline & $415+$ & 455.69 & $46.75(10.3)$ & \\
\hline \multirow[t]{2}{*}{ Ever received a PI } & No & 624.27 & $96.22(15.4)$ & 0.27 \\
\hline & Yes & 3490.92 & $428.98(12.3)$ & \\
\hline \multirow[t]{2}{*}{ Ever received an INSTI } & No & 3689.41 & $504.29(13.7)$ & 0.21 \\
\hline & Yes & 425.78 & $20.92(4.9)$ & \\
\hline
\end{tabular}


Figure 1. Engagement in care during adolescence and young adulthood by CD4 and VL.

\section{Engagement in care (EIC) during adolescence and young adulthood}

(A) Categorised by current $\mathrm{CD} 4$ count

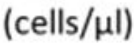

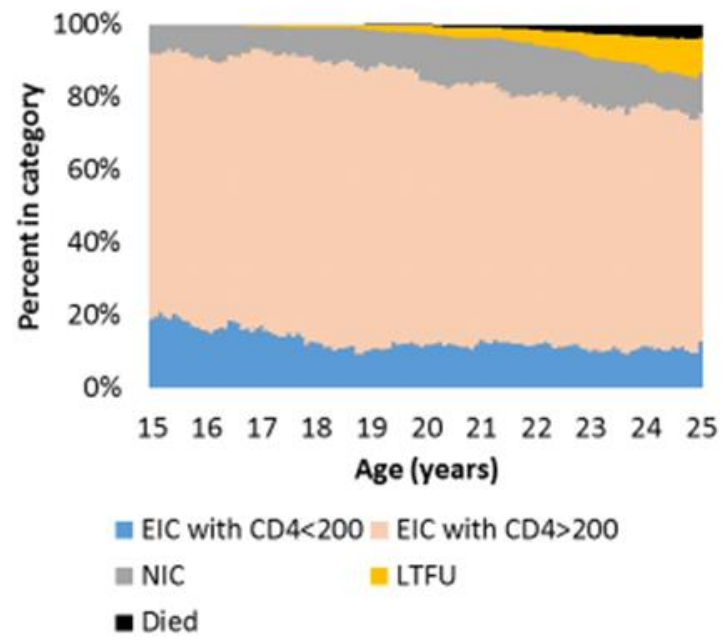

(B) Categorised by current viral load

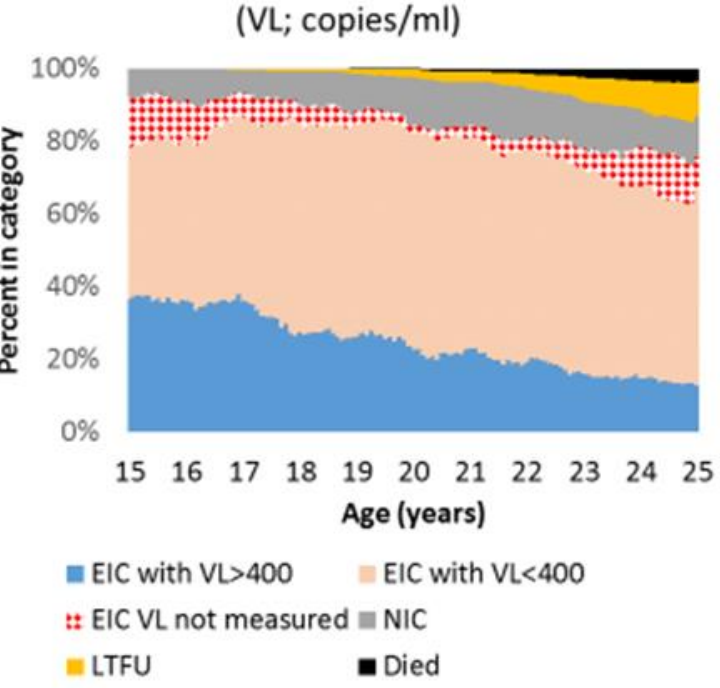

